\title{
Correlation of fluorescence and photocatalytic activity of Co-doped $\mathrm{TiO}_{2}$
}

Pei Wen $\mathrm{Koh}^{1}$, Leny Yuliati ${ }^{2}$, Siew Ling Lee ${ }^{2 *}$

${ }^{I}$ Department of Chemistry, Faculty of Science, Universiti Teknologi Malaysia, 81310 UTM Johor Bahru, Johor, Malaysia.

${ }^{2}$ Centre for Sustainable Nanomaterials, Ibnu Sina Institute for Scientific and Industrial Research, Universiti Teknologi Malaysia, 81310 UTM Johor Bahru, Johor, Malaysia.

*Corresponding Author: sllee@ibnusina.utm.my

Article history :

Received 27 October 2015

Accepted 10 November 2015

\section{GRAPHICAL ABSTRACT}

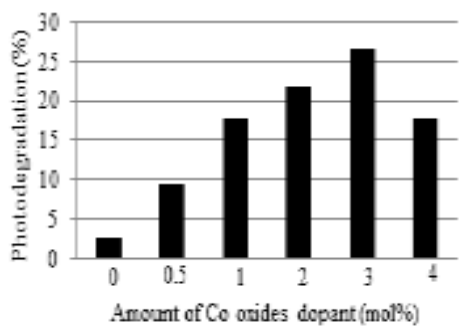

ABSTRACT

Co-doped $\mathrm{TiO}_{2}(0.5-4 \mathrm{~mol} \%)$ photocatalysts were synthesized via sol-gel method and their physical and chemical properties were investigated. Addition of Co oxide dopant induced anatase to rutile phase transition and reduced the band gap energy of $\mathrm{TiO}_{2}$. The fluorescence result indicated that the electronhole recombination rate was reduced with the presence of Co oxide dopant. The best photocatalyst obtained was $3 \mathrm{Co}-\mathrm{TiO}_{2}$ which enhanced the photocatalytic activity of $\mathrm{TiO}_{2}$ by 12 folds to $26.6 \%$. The importance of fluorescence properties of Co-doped $\mathrm{TiO}_{2}$ towards its photodegradation of Congo Red was presented.

Keywords: fluorescence, photocatalysis, Co-doped titania

(C) 2015 Penerbit UTM Press. All rights reserved http://dx.doi.org/10.11113/mjfas.v11n3.382

\section{INTRODUCTION}

Water pollutant is predicted since approximately 1$15 \%$ of the synthetic textile dyes is lost in wastewater streams during manufacturing operations [1]. Hence, it is crucial to treat the wastewater before it is drained to the river and sea. Congo Red (CR) is the first synthetic azo dye and it is widely used in cotton, textile, paper, rubber dyeing industries [2]. CR consists of benzidine structure which is possible to split into carcinogenic amines [3], but it is still widely used in dyeing industry due to its effectiveness and less costly. Hence, removing of CR is an important issue.

Photocatalysis appears to be environmental friendly method since it has the potential to utilize free solar light, operate at room temperature and does not generate secondary pollution compare to other conventional dye removal method including adsorption, filtration, oxidation etc. Metal oxide especially transition metal oxide have been widely attempted to improve the photocatalytic activity of $\mathrm{TiO}_{2}$ under visible light irradiation [4]. Co oxide have been used to improve the photocatalytic activity of $\mathrm{TiO}_{2}$ by various researchers $[5,6]$.

In the previous paper [7], we have demonstrated that Co-doped $\mathrm{TiO}_{2}$ enhanced the photodegradation of Congo Red. It has been proposed that presence of $\mathrm{Co}^{3+}$ might play the role in capturing the electron leading to reduced electron-hole recombination rate. In the current study, additional characterization results were presented and highlighted the correlation between fluorescent study and the photodegradation of $\mathrm{CR}$.

\section{EXPERIMENTS}

\subsection{Photocatalyst Preparation}

The method to synthesize 0.5 to $4 \mathrm{~mol} \%$ Co-doped $\mathrm{TiO}_{2}$ had been reported [7]. In a typical synthesis, precalculated cobalt (II) acetylacetonate (99\%, Acros) dissolved in acetylacetone ( $\geq 99 \%$, Aldrich) was added dropwise to titanium oxide $\left(\mathrm{TiO}_{2}\right)$ precursor which was prepared by mixing titanium tetraisopropoxide $(97 \%$, Aldrich), ethanol $(99.98 \%, \mathrm{HmBG})$ and acetylacetone in the molar ratio 1: 100:2. The mixture was subjected for stirring at room temperature for 30 minutes, followed by evaporation and drying and calcination at $500{ }^{\circ} \mathrm{C}$ for 5 hours.

\subsection{Characterization}

The crystalline structure of the catalyst was determined with X-ray diffraction (XRD) recorded on a 
powder diffractometer (Bruker Advance D8, $40 \mathrm{kV}, 40$ $\mathrm{mA}$ ) using a $\mathrm{Cu} \mathrm{K} \alpha$ radiation source in the range of $2 \theta=$ $20-80^{\circ}$ with scan rate of $0.1 \%$. The rutile phase was calculated using Spurr equation as shown in equation (1).

$\mathrm{R}(\%)=100 /\left(1+0.8\left[\mathrm{I}_{\mathrm{A}} / \mathrm{I}_{\mathrm{R}}\right]\right)$

where $\mathrm{I}_{\mathrm{A}}$ and $\mathrm{I}_{\mathrm{R}}$ are the intensity values of the anatase at $2 \theta$ $=25.3^{\circ}(101)$ and rutile peak at $2 \theta=27.5^{\circ}(110)$. Diffuse reflectance UV-Visible spectra of samples were recorded on Perkin Elmer Lambda 900 Ultraviolet-visible spectrometer with $\mathrm{BaSO}_{4}$ as reference. Band gap energy was obtained from Tauc plot which is the plot of $(\alpha h v)^{2}$ against $h v$ where $\alpha$ is the absorbance obtained from diffuse reflectance UV-Vis spectrum, $h$ is the Planck constant and $v$ is the frequency of light. $h v$ can be calculated as $1240 /$ wavelength. The band gap value was determined by extrapolating the vertical segment of the plot to intersect on the $\mathrm{x}$-axis when $\mathrm{y}$-axis is zero. The fluorescence spectra of the photocatalysts $(2.000 \pm 0.005 \mathrm{~g})$ were recorded by a spectrofluorometer (JASCO, FP-8500) with an excitation wavelength at $216 \mathrm{~nm}$.

The photocatalytic activity of the samples was tested via photodegradation of $\mathrm{CR}$ under visible light irradiation using halogen fiber optic light illuminator (Dolan-Jenner MI 157, $150 \mathrm{~W})$. UV longpass filter (400 nm, Edmund Optics) was used to allow only visible light $(\lambda>400 \mathrm{~nm})$ irradiated on the dye solution. Sample $(0.1 \mathrm{~g})$ was put into the beaker consisted of $50 \mathrm{~mL} 100 \mathrm{ppm}$ of $\mathrm{CR}$ and subjected to light illumination for 24 hours. Adsorption test was carried out prior to photocatalytic study to ensure equilibrium has been achieved. After the photocatalytic reaction, the solution was centrifuged and the supernatant was withdrawn. The concentration of $\mathrm{CR}$ was determined using UV-Vis spectrometer (Thermo Fisher, Genesys 10S) at $\lambda=499 \mathrm{~nm}$. The photocatalytic activity was examined by determining the decolorization percentage of $\left(\mathrm{C}_{0}-\mathrm{C}\right) / \mathrm{C}_{0}$ of $\mathrm{CR}$ where $\mathrm{C}_{0}$ and $\mathrm{C}$ were the concentration of $\mathrm{CR}$ before and after the photocatalytic reaction, respectively.

\section{RESULTS AND DISCUSSION}

The ability of cobalt oxide dopant to induce anatase to rutile phase transformation was investigated and the XRD pattern is depicted in Figure 1. The percentage of antase and rutile phase is shown in Table 1. It was found out that it required up to $4 \mathrm{~mol} \%$ Co oxide dopant to induced anatase to rutile phase transformation. The ionic size difference between $\mathrm{Ti}^{4+}(0.605 \AA)$ and $\mathrm{Co}^{2+}(0.65 \AA)$, and $\mathrm{Co}^{3+}(0.545 \AA)$ is less than $15 \%$, thus substitution of $\mathrm{Ti}^{4+}$ with either $\mathrm{Co}^{2+}$ or $\mathrm{Co}^{3+}$ is possible which lead to anatase to rutile phase transformation [8]. The added Co oxide could have initially incorporated in the interstitial position of $\mathrm{TiO}_{2}$ framework when the amount of Co oxide was lower than $4 \mathrm{~mol} \%$ and the additional Co oxide dopant could have incorporated inside $\mathrm{TiO}_{2}$ framework in the substitutional position when $4 \mathrm{~mol} \%$ Co oxide were doped. The crystallite size was calculated using Scherrer equation. Obviously, crystallite size of $\mathrm{TiO}_{2}$ was reduced from 20.10 to $15.76 \AA$ when doped with $0.5 \mathrm{~mol} \%$ of Co oxide dopant. This indicated that $\mathrm{Co}$ oxide could inhibit the crystal growth of $\mathrm{TiO}_{2}$. Further addition of Co oxide has negligible effect on the crystallite size of $\mathrm{TiO}_{2}$.

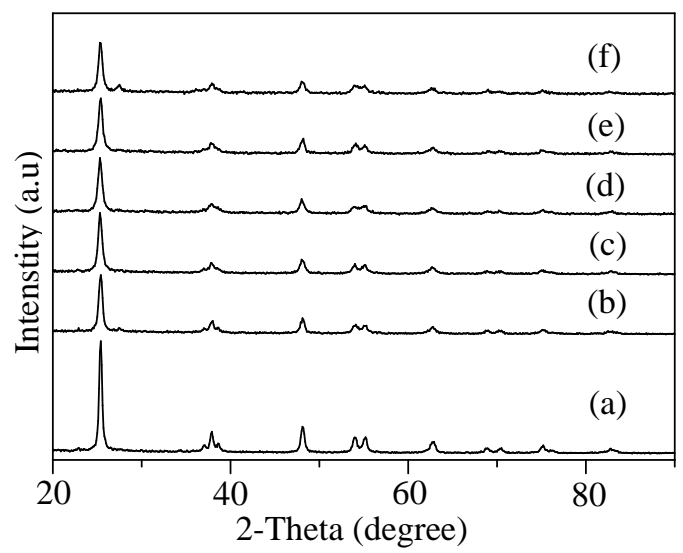

Fig. 1 XRD pattern of (a) $\mathrm{TiO}_{2}$, (b) $0.5 \mathrm{Co}-\mathrm{TiO}_{2}$, (c) $1 \mathrm{Co}-\mathrm{TiO}_{2}$, (d) $2 \mathrm{Co}-\mathrm{TiO}_{2}$, (e) $3 \mathrm{Co}-\mathrm{TiO}_{2}$, (f) $4 \mathrm{Co}-\mathrm{TiO}_{2}$

Band gap energy is a crucial factor which determines if the sample is visible-light-driven photocatalyst. The band gap energies of the samples are shown in Table 1. Band gap energy of $\mathrm{TiO}_{2}$ was successfully reduced by $42 \%$ from 3.25 to $2.35 \mathrm{eV}$ when doped with $4 \mathrm{~mol} \%$ Co oxide, indicating that the optical properties of $\mathrm{TiO}_{2}$ has been successfully changed from UV active to visible light active with Co oxide doping. Since the standard reduction potential of $\mathrm{Co}^{2+} / \mathrm{Co}^{3+}$ redox couple is $1.92 \mathrm{eV}$ [9], the added Co oxide dopant can create additional energy level below the conduction band of $\mathrm{TiO}_{2}$. In addition, when the amount of Co oxide dopant increase, the density of state of the dopant increase and form a continuum of states just like the bands and effectively the band gap energy decreased.

The fate of electron and hole recombination rate was studied via fluorescence analysis. The fluorescence spectra of $\mathrm{TiO}_{2}$ and Co-doped $\mathrm{TiO}_{2}$ are illustrated in Figure 2. The undoped $\mathrm{TiO}_{2}$ and all Co-doped $\mathrm{TiO}_{2}$ samples have similar emission spectra. Three peaks at 407, 467, and $553 \mathrm{~nm}$ were detected for all the samples. The first peak was corresponded to emission of band gap transition while the other two peaks were attributed to charge-transfer of oxygen vacancy trapped electron [10]. As can be seen, the peak intensity of $\mathrm{TiO}_{2}$ reduced significantly after addition of $0.5 \mathrm{~mol} \%$ Co oxide dopant. The peak intensity continually decreased with further increment of Co oxide dopant up to $3 \mathrm{~mol} \%$. Since the fluorescence emission is the result of recombination of excited electrons and holes, the lower emission intensity of the Co-doped $\mathrm{TiO}_{2}$ inferred to a lower recombination rate. In other words, $3 \mathrm{Co}-\mathrm{TiO}_{2}$ has the lowest electrons and holes recombination rate among the prepared Co-doped $\mathrm{TiO}_{2}$ samples. 
Koh et al. / Malaysian Journal of Fundamental and Applied Sciences Vol. 11, No. 3 (2015) 126-129

Table 1 Rutile phase, crystallite size, band gap energy, surface area and adsorption ability of samples

\begin{tabular}{ccccc}
\hline Sample & Rutile phase (\%) & Crystallite size (nm) & Band gap energy (eV) & Adsorption (\%) \\
\hline $\mathrm{TiO}_{2}$ & 0 & 20.10 & 3.25 & 0.9 \\
$0.5 \mathrm{Co}-\mathrm{TiO}_{2}$ & 0 & 15.76 & 3.20 & 4.4 \\
$1 \mathrm{Co}-\mathrm{TiO}_{2}$ & 0 & 15.54 & 3.15 & 4.9 \\
$2 \mathrm{Co}-\mathrm{TiO}_{2}$ & 0 & 14.58 & 2.85 & 9.9 \\
$3 \mathrm{Co}-\mathrm{TiO}_{2}$ & 0 & 15.19 & 2.55 & 10.4 \\
$4 \mathrm{Co}-\mathrm{TiO}_{2}$ & 22 & 15.45 & 2.35 & 11.5 \\
\hline
\end{tabular}

The reduced recombination rate could be attributed to the presence of $\mathrm{Co}^{3+} / \mathrm{Co}^{2+}$ redox couple [11]. On the other hand, it was found that further loading of Co oxide into/onto $\mathrm{TiO}_{2}$ has caused increment in peak intensity in the emission spectrum as evidenced in $4 \mathrm{Co}-\mathrm{TiO}_{2}$ sample. The finding may imply that $4 \mathrm{~mol} \%$ Co oxide dopant was in excess and hence they acted as the recombination centre. As a result, the electron and hole recombination rate of $4 \mathrm{Co}-\mathrm{TiO}_{2}$ was slightly higher than that of $3 \mathrm{Co}-\mathrm{TiO}_{2}$. Furthermore, it was reported that rutile phase has more defect and hence led to greater electron-hole recombination rate [12].

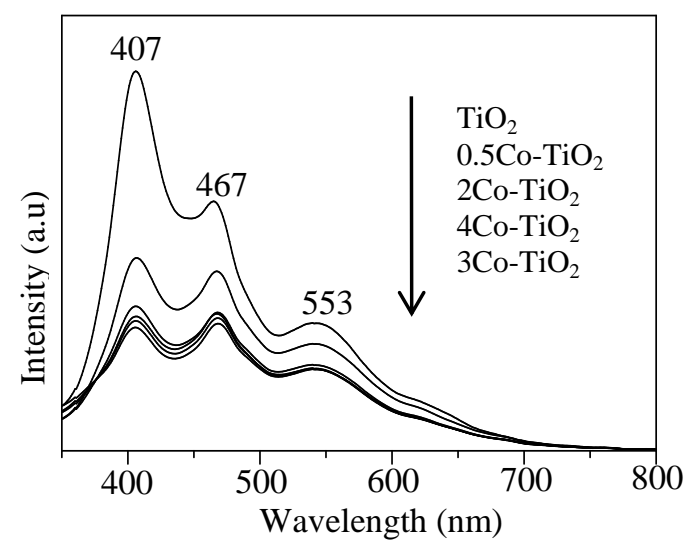

Fig. 2 Fluorescence spectra of $\mathrm{TiO}_{2}$ and Co-doped $\mathrm{TiO}_{2}$ samples

The photodegradation of $50 \mathrm{~mL} 100 \mathrm{ppm}$ CR was tested over the prepared Co-doped $\mathrm{TiO}_{2}$. Adsorption test was carried out in the dark before the reaction was carried out under visible light illumination. Results (Table 1) showed that the adsorption of dye on the photocatalysts increased with increasing of Co oxide dopant amount. After 2 hours, 4.4, 4.9, 9.9, 10.4 and $11.5 \%$ of CR were adsorbed on $0.5 \mathrm{Co}-\mathrm{TiO}_{2}, 1 \mathrm{Co}-\mathrm{TiO}_{2}, 2 \mathrm{Co}-\mathrm{TiO}_{2}, 3 \mathrm{Co}-\mathrm{TiO}_{2}$, and $4 \mathrm{Co}-\mathrm{TiO}_{2}$, respectively. This phenomenon can be visualized by increasing of electrostatic charge interaction between cationic charge of $\mathrm{Co}$ ions on Co-doped $\mathrm{TiO}_{2}$ samples and anionic charge of CR.

The results of photodegradation of $\mathrm{CR}$ are shown in Figure 3. It was demonstrated that addition of Co oxide enhanced remarkably the photocatalytic activity of $\mathrm{TiO}_{2}$. The loading of cobalt oxide into/onto $\mathrm{TiO}_{2}$ has gradually increased the photodegradation activity of $\mathrm{CR}$, from $2.3 \%$ by undoped $\mathrm{TiO}_{2}$ up to $26.6 \%$ by $3 \mathrm{Co}-\mathrm{TiO}_{2}$ photocatalyst.
The increment was about 12 folds. The enhancement of the photocatalytic activity could be due to extension of absorption edge of $\mathrm{TiO}_{2}$ to the visible light region which enabled utilization of visible light as activation source. In addition, the increment of electrostatic interaction between $\mathrm{CR}$ and Co-doped $\mathrm{TiO}_{2}$ photocatalyst could also improve photocatalytic activity since photocatalysis is a surface reaction and the targeted pollutant must contact with the photocatalyst first for the degradation process to take place. The reduction of electrons and holes recombination rate as a result of the presence of $\mathrm{Co}^{3+} / \mathrm{Co}^{2+}$ redox couple also led to the enhanced photodegradation of CR. Rather than extended absorption in visible region and reduced band gap energy, our research findings suggested that low electrons and holes recombination rate was the key factor for high photocatalytic activity. Amount of $3 \mathrm{~mol} \%$ was the optimum Co oxide dopant amount to enhance the photodegradation of $\mathrm{CR}$ due to its lowest electrons and holes recombination rate. On the other hand, excess of Co oxide dopant in $4 \mathrm{Co}-\mathrm{TiO}_{2}$ hampered the activity due to over dosage. It was believed that the excess Co oxide acted as electrons and holes recombination center.

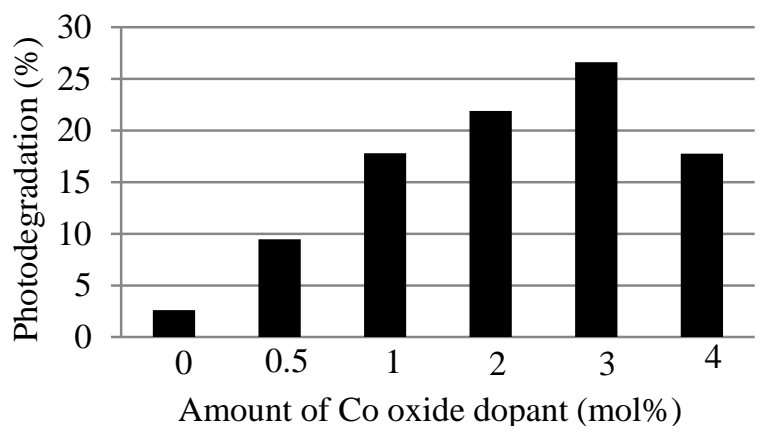

Fig. 3 Photodegradation of Congo Red by 0.5 to $4 \mathrm{~mol} \% \mathrm{Co}-$ doped $\mathrm{TiO}_{2}$. (concentration of $\mathrm{CR}=100 \mathrm{ppm}$, time $=24$ hours, room temperature)

\section{CONCLUSION}

The current study indicated that the variation photodegradation of $\mathrm{CR}$ by $\mathrm{Co}$-doped $\mathrm{TiO}_{2}$ as a function of amount of Co oxide was closely related to the electron-hole recombination rate. Factors including presence of rutile phase, crystallite size, band gap energy and adsorption ability did not affect directly the photocatalytic behavior of Co-doped $\mathrm{TiO}_{2}$ photocatalyst under visible light irradiation. 


\section{ACKNOWLEDGEMENTS}

The authors gratefully acknowledge the Ministry of Education Malaysia (MOE) and Universiti Teknologi Malaysia (UTM) for the Fundamental Research Grant Scheme (FRGS, vote no.: R.J. 130000.7809.4F527).

\section{REFERENCES}

[1] H. Zhu, R. Jiang, L. Xiao, Y. Chang, Y. Guan, X. Li, G. Zeng, J Hazard Mater 169 (2009) 933.

[2] Z. L. Yaneva, N. V. Georgieva, Int. Rev. Chem. Eng. 4 (2012) 127.

[3] A. Pielesz, A. Weselucha-Birczynska, J. Mol. Struc. 555 (2000) 325 .

[4] A. Zaleska, Recent Patents on Engineering 2 (2008) 157

[5] C. T. Hsieh, W. S. Fan, W. Y. Chen, J. Y. Lin, Sep. Purif. Technol. 67 (2009) 312.

[6] J. J. Li, S. Q. Liu, Y. Y. He, J. Q. Wang, Micropor. Mesopor. Mater. 115 (2008) 416.

[7] P. W. Koh, L. Yuliati, S. L. Lee, J. Teknologi 69 (2014) 45.

[8] R. Arroyo, G. Cordoba, J. Padilla, V. H. Lara, Mater. Lett. 54 (2002) 397.

[9] A. J. Bard, R. Parsons, J. Jordan, Standard Potentials in Aqueous Solutions, CRC Press, Marcel Dekker, New York, 1985.

[10] Y. Cong, J. Zhang, F. Chen, M. Anpo, J. Phys. Chem. C 111 (2007) 6976.

[11] F. Xia, E. Ou, L. Wang, J. Wang, Dyes. Pigm. 76 (2008) 76.

[12] A. Sclafani, J. M. Herrmann, J. Phys. Chem. 100 (1996) 13655. 